\title{
An Analysis of energy efficient Homogeneous WSN protocols
}

\author{
Shipra Singla \\ Asst. professor, Computer science and Engineering, \\ Giani Zail Singh College of Engineering, Bathinda, India \\ Jabarweer Singh \\ Asst. professor, Computer science and Engineering, \\ Giani Zail Singh College of Engineering, Bathinda, India
}

\begin{abstract}
This paper is a review on the critical problem of network lifetime of WSNs. Due to limited battery of sensor nodes, so energy efficiency found to be main constraint of limited life of WSNs. Therefore the main focus of the present work is to find the ways to minimize the energy consumption problem and how one can enhance the network stability period and life time. Many researchers have proposed different kind of the protocols to enhance the network lifetime but still much improvement can be done further to enhance the network lifetime further. The overall objective of this paper is to evaluate the gaps in existing clustering techniques of WSNs. This paper has evaluated the issues which have been neglected in the field of the WSNs.
\end{abstract}

Keywords - WSNs, Network Lifetime, Stable PERIOd, Clustering, LeaCH, DeEC.

\section{INTRODUCTION}

Wireless Sensor Networks (WSNs) are networks that consist of nodes also called sensors which are deployed in a region. These sensors work with each other to sense various types of physical information from the atmosphere. In various significant fields WSNs are very helpful like environmental traffic, military surveillance, area monitoring, air pollution monitoring, wastewater monitoring, pressure etc [1] [9]. Current WSNs is working on the problems of low-power communication, computation and energy storage.

All sensor nodes process data and transmit it to base station also called sink. In WSNs nodes are battery constrained due to limited energy [2] [3] [4]. So use of the battery in efficient way becomes critical issue. A number of protocols play an important role to reduce energy consumption. Direct communication and multi-hop data transmission used initially. But due to limited power of sensor nodes these techniques don't work effectively.

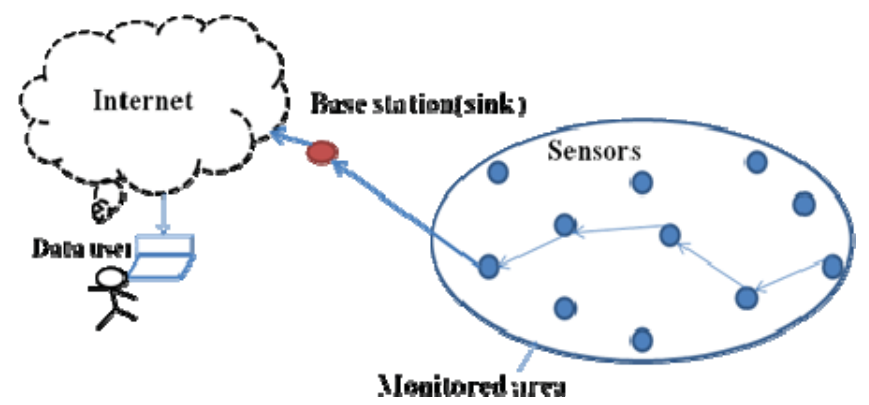

Fig.1. Wireless sensor network

Energy is very critical issue in WSN, because of limited energy in sensor nodes, so to conserve energy clustering technique was introduced; in which out of thousands of nodes few nodes become cluster head and they manage the entire network.

Cluster head is a node which is responsible for maintain cluster, collect data from nodes in the cluster and communicating with sink. By using clustering methodology it has been observed that there is large amount of 
energy that has been saved. In static clustering method some rules were followed to elect a cluster head, once a cluster is formed and cluster head is elected, the cluster was statically operated until the head node dead.

Because cluster head node have more responsibility so rapid decrease in energy in the Cluster head node. The death time was head node was too early in static clustering technique. So there was a need required the Wenzimen proposed a protocol based on adaptive clustering technique he named it LEACH.

\section{CLUSTERING}

Clustering [2] [4] [6] [9] is a technique where nodes are arranged into clusters that are useful in achieving energy efficiency. All nodes belonging to the same cluster send their data to cluster head. The main function of cluster head is to provide efficiently data communication between sensor nodes and the base station. So the cluster head should have high energy as compared to other nodes. $\mathrm{CH}$ aggregates data and sends aggregated data to BS where the enduser can access the data.

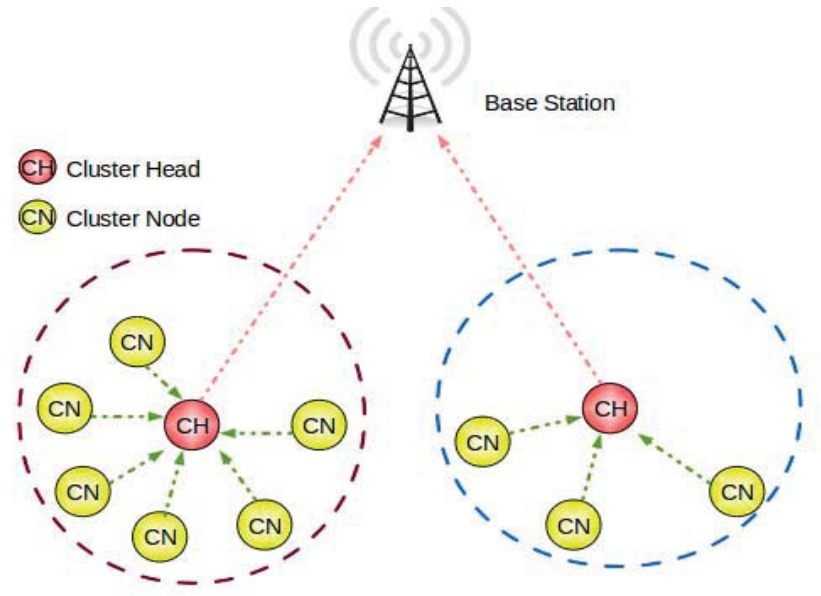

Fig.2. Cluster formation in WSN [1]

\section{III.DIFFERENT CLUSTERING SCHEMES}

In Wireless sensor network clustering can be done in two types of network i.e. homogeneous and heterogeneous. All sensor nodes with identical energy level are known as homogeneous WSNs. Low-Energy Adaptive Clustering Hierarchy (LEACH) [4],

Threshold sensitive energy efficient sensor network protocol (TEEN) [5], LEACH-centralized (LEACH-c) [6], Adaptive threshold sensitive energy efficient sensor network protocol (APTEEN) [7], Power Efficient Gathering in Sensor Information Systems (PEGASIS) [8], Hybrid Energy-Efficient Distributed Clustering (HEED) [9] protocols are widely used for homogeneous WSNs.

In heterogeneous WSNs all sensor nodes have dissimilar energy level and fewer energy nodes died first than the high energy sensor nodes. Stable Election Protocol (SEP) [10], Distributed Energy Efficient Clustering (DEEC) [11], Developed DEEC (DDEEC) [15] and Enhanced DEEC (EDEEC) [16] are well known heterogeneous WSNs protocols. Low-Energy Adaptive Clustering Hierarchy (LEACH) [4] used for homogeneous protocol in which all the nodes have same energy level. There are number of rounds for communication of information. Each round starts with set-up phase and followed by the steady phase. 


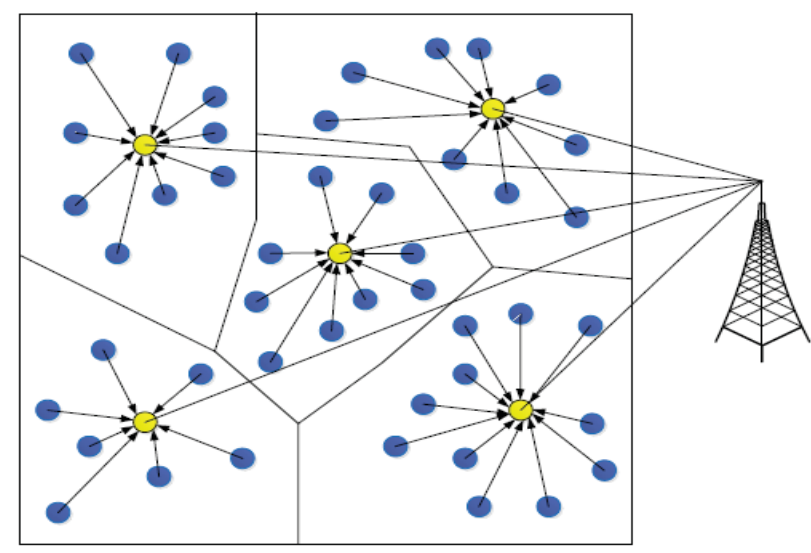

Fig.3. LEACH Clustering Hierarchy [3]

In first phase i.e. setup phase cluster creation and $\mathrm{CH}$ selection was take place. $\mathrm{CHs}$ selection choice is prepared by the each node select a random number between 0 and 1 . Threshold $\mathrm{T}(\mathrm{n})=\mathrm{P} /\{1-\mathrm{P}(\mathrm{r} \bmod 1 / \mathrm{P})\}$

calculated to check a node has chance to become $\mathrm{CHs}$ for current round. In this $\mathrm{P}$ is desire percentage of CHs, $\mathrm{r}$ is the number of current round. If node contains value less than $\mathrm{T}(\mathrm{n})$ it becomes $\mathrm{CHs}$ for current round and cannot be $\mathrm{CHs}$ for the next1 / P rounds. Therefore probability of remaining nodes must be increased. After this in steady phase CHs node receives all data from local nodes compress it and send it to the sink. LEACH is an effective technique to reduce energy dissipation, enhanced network lifetime. Distributed Energy Efficient Clustering (DEEC) [11] is used for heterogeneous WSNs. In DEEC, the CHs chosen by a possibility based on the ratio between the remaining energy of every node and the average energy of the WSNs. The round number of the rotating period for every sensor node is dissimilar to its initial and remaining energy. The sensor nodes with maximum initial and remaining energy will have more chance to become the CHs than normal nodes. In LEACH every node has chance to become a CHs after $1 / \mathrm{p}$ rounds. All the nodes cannot same remaining energy when sensor network evolves so, the energy will be not well distributed and the low-energy nodes will finish earlier than the high-energy nodes. For CH choice, DEEC uses initial and remaining energy level of nodes. DEEC provides good performance in the networks containing normal and advanced nodes. Developed Distributed Energy-Efficient Clustering (DDEEC) [15] allows to balance the cluster head selection overall WSNs nodes following their remaining energy. DDEEC uses same method for estimation of average energy and CH selection algorithm based on remaining energy as applied in DEEC[22]. In DDEEC nodes that have maximum energy values and more remaining energy has more possibility to become $\mathrm{CH}$ than nodes having lower energy, so, in these way advanced nodes will become CHs more often as compared to normal nodes. After some period advanced nodes having same remaining energy like normal nodes. Although, DEEC continues to punish the advanced nodes so this is not best way for energy distribution, because after this advanced nodes are continuously be a $\mathrm{CH}$ and they expire faster than normal nodes. To avoid this problem DDEEC presents a threshold residual energy. When advanced and normal nodes energy level less than threshold residual energy then both types of nodes use same probability to become cluster head[33]. Therefore, $\mathrm{CH}$ selection is balanced and more efficient in DDEEC. Enhanced Distributed Energy Efficient Clustering (EDEEC) [16] scheme uses the idea of DEEC with addition of super nodes and expands it into three level heterogeneity WSNs. It includes three types of nodes i.e. normal, advanced and super nodes with their probabilities based on initial energy. Enhanced Developed Distributed Energy Efficient Clustering (EDDEEC) [2] scheme is used for three-level heterogeneous WSNs. It uses same method for $\mathrm{CH}$ selection based on initial, remaining energy level of the nodes and average energy of network as in DEEC. In EDEEC after some rounds, some super and advance nodes have same remaining energy level as normal nodes due to continually $\mathrm{CH}$ selection.[20] Therefore it continues to penalize advance and super sensor nodes. Same issue with DEEC, it also continues to penalize just advance nodes and DDEEC is limited only for two-level heterogeneous networks. To remove this unbalanced problem in three-level heterogeneous WSNs EDDEEC changes in function which described in EDEEC for calculating probabilities of normal, advance and super nodes[22]. These modifications are based on absolute remaining energy level that is the value in which advance and super sensor nodes have similar energy level as in case of normal nodes. Using absolute remaining energy all kinds of nodes has identical probability for $\mathrm{CH}$ selection.

\section{RELATED WORK}


Heinzelman et al. (2000) [4] has proposed Low-Energy Adaptive Clustering Hierarchy (LEACH) protocol for homogeneous WSNs that has all nodes of same energy level. In LEACH every node to become a cluster head depends on its individual probability. It equally assigned the energy load among the sensor nodes by use of randomized alternation of cluster-heads. Manjeshwar et al. (2001) [5] has introduced a new protocol TEEN (Threshold sensitive energy efficient sensor network protocol) in which the cluster head uses two values of threshold, hard threshold and soft threshold. Hard threshold is the minimum value of the attribute that activates the communication from node to the cluster head and soft threshold is small change in the value of the sensed attributes. This technique reduced the number of transmission. Chandrakasan et al. (2002) [6] has proposed LEACHCentralized protocol. LEACH did not provide the guarantee about the position and number of group leader nodes. So LEACH-C is more efficient because in this base station has global information of the energy level and location of all the nodes, so it created better clusters. Manjeshwar et al. (2002) [7] has presented Adaptive Threshold sensitive Energy Efficient sensor Network protocol also called APTEEN. It extended the rules of Teen which is a hybrid protocol for both periodic up data collection and also for time significant data collection. It offered data regularly and also supplied information on time significant actions. It combined the best feature of both proactive and reactive network. Lindsey et al. (2002) [8] has presented an improved scheme Power-Efficient Gathering in Sensor Information Systems also called PEGASIS. PEGASIS is a chain-based protocol instead of cluster-based protocol. Each node communicates only with its neighbor node and transmitting data to the sink, so it reduced the amount of energy spent per cycle. Youniset al. (2004) [9] has presented a homogeneous protocol Hybrid Energy-Efficient Distributed Clustering (HEED). It extended the scheme of LEACH algorithm by including remaining energy. This method balanced the load on sensor nodes and gave better improvement in lifetime of the network It offered the guarantee that the maximum energy node will be the cluster head inside its cluster range. Smaragdakis et al. (2004) [10] has proposed Stable Election Protocol also called SEP. It was made for the two-level heterogeneous networks that contain two types of sensor nodes. According to their own energy each node is able to become cluster head, based on individual selection probability. SEP improved the stability time, which is defined as the time period before death of the first node. Qing et al. (2006) [11] has presented a new protocol Distributed energy-efficient clustering (DEEC) for heterogeneous WSNs. Cluster-heads selection depends on the ratio between remaining energy of each node and the average energy of WSNs. In this protocol, node containing more energy has more probability to be a cluster head. DEEC does not need full energy information at every time though the choice of cluster head. Israr et al. (2007) [12] has presented an improved protocol Multi-hop LEACH that carried out intra-cluster transmission in which sensor nodes send data to its cluster head and inter-cluster transmission hold temporary cluster head. It improved the load balancing problem and is more efficient in terms of energy utilization from LEACH and LEACH-C. Ali et al. (2008) [13] has proposed an improved protocol advanced low-energy adaptive clustering hierarchy (ALEACH). It depends on both the present status probability and general probability that enables selecting well suitable node for cluster head and rotating cluster head positions to evenly distribute the energy load among all the nodes. Elbhiri et al. (2009) [14] has proposed Distributed Energy Efficient Clustering also called SDEEC for the heterogeneous WSNs. It permits to balance the cluster head choice overall network nodes follow their remaining energy. It has optimized the intra-clusters communication to prolong network lifetime and give better performance than the SEP and DEEC. Elbhiri et al. (2010) [15] has proposed a new heterogeneous protocol called Developed Distributed Energy-Efficient Clustering (DDEEC). This choice of protocol always selected the advanced nodes, when their remaining energy decrease and be converted into the series of the normal sensor nodes. It optimized the cluster head choice by following their remaining energy and it performed better than DEEC. Saini et al. (2010) [16] has presented an improved protocol Enhanced Distributed Energy Efficient Clustering (EDEEC). EDEEC comes up with three kinds of sensor nodes that can be used to increase the lifetime and stability of the WSNs. It enhances the heterogeneity, energy level and received more data packets at BS than SEP. Alla et al. (2011) [17] has proposed a new technique Balanced and Centralized Distributed Energy Efficient Clustering also called BCDEEC. In this BS guarantees that the high energy nodes suitable first entrance for cluster heads to get better lifetime of WSNs and saving average energy It gives the better performance than SEP and DEEC. Miao et al. (2012) [18] has proposed a performance analyses of LEACH, DEEC and SEP. LEACH does not perform well in heterogeneous environment. SEP is composed of two types of nodes according to the initial power. In DEEC High initial and remaining energy nodes will have more probability to be $\mathrm{CH}$ than lower energy nodes. Thus, DEEC can improve the stability period and Lifetime of WSNs. Qureshi et al. (2012) [19] has tested the performance of Distributed Energy- Efficient Clustering, Threshold DEEC, Developed DEEC and Enhanced DEEC. It tested these schemes under a number of different condition hold high level heterogeneity to low level heterogeneity. DEEC and DDEEC performed well in three level heterogeneous network hold high energy level variation among nodes. EDEEC and TDEEC performed well in all heterogeneous state containing low energy level variation among. S. Pal et al.(2004) [20] has witnessed a lot of attention in routing for wireless sensor networks and 
introduced unique challenges compared to traditional data routing in wired networks. Routing in sensor networks is a new area of research.In this first the author have gone through a comprehensive survey of routing techniques in wireless sensor networks. The routing techniques are classified as proactive, reactive and hybrid on their mode of function and type of target applications. In this eight routing protocols and their comprehensive survey is discussed. These eight protocols are LEACH, TEEN, APTEEN, PEGASIS, SPIN, DD, RR and GEAR. Since the sensor networks are application specific, we can't say a particular protocol is better than other. comparison of these protocols only on the basis of parameters is done.Future perspective of this work is well focused towards modifying one of the above routing protocols such that the modified protocol could minimize more energy for the entire system. Kashaf et al.(2012) [21] has proposed a new protocol Threshold Sensitive Stable Election Protocol (TSEP), which is reactive protocol using three levels of heterogeneity. Reactive netwroks, as opposed to proactive networks, respond immediately to changes in relevant parameters of interest. The performance of new protocol for a simple temperature sensing application and compare results of protocol with some other protocols LEACH, DEEC, SEP,ESEP and TEEN. And from results it is observed that protocol outperforms concerning life time of sensing nodes used. M.Vivek et.al [22] (2004) has presented cost based comparative study of homogeneous and heterogeneous clustered sensor networks. He focused on the case where the base station is remotely located and the sensor nodes are not mobile. Since we are concerned with the overall network dimensioning problem, we take into account the manufacturing cost of the hardware as well as the battery energy of the nodes. A homogeneous sensor network consists of identical nodes, while a heterogeneous sensor network consists of two or more types of nodes (organized into hierarchical clusters). We first consider single hop clustered sensor networks (nodes use single hopping to reach the cluster heads). We use LEACH as the representative single hop homogeneous network, and a sensor network with two types of nodes as a representative single hop heterogeneous network. For multi-hop homogeneous networks (nodes use multi-hopping to reach the cluster head), we propose and analyze a multi-hop variant of LEACH that we call M-LEACH. We show that M-LEACH has better energy efficiency than LEACH in many cases. We then compare the cost of multi-hop clustered sensor networks with M-LEACH as the representative homogeneous network, and a sensor network with two types of nodes (that use in-cluster multi-hopping) as the representative heterogeneous network. He showed that in many cases M-LEACH is more energy efficient than LEACH. Using M-LEACH as the representative multi-hop homogeneous network, we presented a cost based comparison of multi-hop homogeneous and multihop heterogeneous networks.

\section{COMPARISON AMONG DIFFERENT ROUTING PROTOCOLS}

TABLE I COMPARISON AMONG DIFFERENT ROUTING PROTOCOLS

\begin{tabular}{|l|l|l|l|l|l|}
\hline PROTOCOLS & $\begin{array}{l}\text { POWER } \\
\text { MANAGEMENT }\end{array}$ & $\begin{array}{l}\text { N/W } \\
\text { LIFETIME }\end{array}$ & CLASSIFICATION & MULTIPATH & SCALABILITY \\
\hline LEACH & Maximum & Very good & Clustering & No & Good \\
\hline TEEN & Maximum & Very good & Clustering/Reactive & No & Good \\
\hline APTEEN & Maximum & Very good & Hybrid & No & Good \\
\hline PEGASIS & Maximum & Very good & Reactive/Clustering & No & Good \\
\hline SPIN & Limited & Good & Proactive/Flat & Yes & Limited \\
\hline DD & Limited & Good & Proactive/Flat & Yes & Limited \\
\hline GEAR & Limited & Good & Location & No & Limited \\
\hline
\end{tabular}

Now we compare the above mentioned routing protocols according to their performance depending on different parameters. The Table I shows the comparison and show that LEACH, TEEN, APTEEN, PEGASIS - they have similar features and their architectures are to some extent similar. They have fixed infrastructure. LEACH, TEEN, APTEEN are cluster based routing protocols where as PEGASIS is chain-based protocol. The performance [4] of APTEEN lies between TEEN and LEACH with respect to energy consumption and longevity of the network. TEEN only transmits time critical data where as APTEEN perform periodic data transmission. In this respect APTEEN is 
also better than LEACH because APTEEN transmits data based on the threshold value where as LEACH transmits data continuously. Again PEGASIS avoids the forming of clustering overhead of LEACH, but it requires dynamic topology adjustment since sensor's energy is not tracked. PEGASIS introduces excessive delay for distant node on the chain. The single leader can become a bottleneck in PEGASIS. PEGASIS increases network lifetime twice as compared to LEACH protocol. In directed diffusion base station sends queries to sensor nodes by flooding technique but in SPIN the sensor nodes advertises the availability of data so that interested nodes can query on that data. In Directed diffusion each node can communicate with its neighbors; so it does not need the total network information. But SPIN maintains a global network topology. SPIN halves the redundant data in comparison to flooding. Since SPIN cannot guarantee data delivery it is not suitable for applications that need reliable data delivery SPIN, directed diffusion and rumor routing use meta-data where as the other protocols don't use it. As they are flat routing protocols routes are formed in regions that have data for transmission. But for the others, as they are hierarchical routing they forms clusters throughout the network. In case of hierarchical routing energy dissipation is uniform and it can't be controlled ; but in case of flat routing energy dissipation depends on the traffic pattern. For the previous case data aggregation is done by cluster head but in the later case, nodes on multihop path aggregates incoming data from neigbours. GEAR limits the number of interests in Directed Diffusion by considering only a certain region rather than sending the interests to the whole network. GEAR thus complements Directed Diffusion and conserves more energy. Since the sensor networks are application specific, we can't say a particular protocol is better than other.

\section{GAPS IN LITERATURE}

By conducting the literature survey it has been found that the every WSNs protocol has some limitations; i.e. no one is perfect in every case and most of the existing literature has neglected one of the following:

1. The Most of the existing researchers has neglected the use of the distance between the sensor node and the base station while selecting the cluster head.

2. The optimum numbers of clusters in every round are not consistent in LEACH as well as in DEEC variants.

\section{ANALYTICAL SOLUTION}

One can improve the performance of the EDDEEC using fuzzy based cluster head selection. The EDDEEC has used different probability function for selecting the best cluster head by using the residual energy and average energy of the network. But EDDEEC has neglected the distance between base station and cluster. The fuzzy cost will be evaluated on the basis of the residual energy and the node centrality. The fuzzy cost will be dynamic in nature as it is evaluated in each round. Thus will provide more better results due to its adaptive nature i.e. will change as the residual energy changes. The main advantage of this solution is the optimum numbers of clusters are formed in every round, which is almost impossible in LEACH and also not guaranteed in EDDEEC.

\section{CONCLUSION}

It has been found from the survey that the most of the existing researchers has worked hard to prolong the network lifetime. This has come up with significant improvement over the existing protocols like LEACH. But it is also found that the most of the researchers has neglected at least one of these issues of WSNs. (a) Most of the researchers has neglected Fuzzy based cluster head selection. (b) The Most of the existing researchers has neglected the use of the distance between the sensor node and the base station while selecting the cluster head. (c) The optimum numbers of clusters in every round are not consistent in LEACH as well as in DEEC variants.

In near future we will use fuzzy cost which will be evaluated on the basis of the residual energy and the node centrality. The fuzzy cost will be dynamic in nature as it will be evaluated in each round. Thus will provide more better results due to its adaptive nature i.e. will change as the residual energy changes. The main advantage of this suggested protocol is that the optimum numbers of clusters will be formed in every round, which is almost impossible in LEACH and also not guaranteed in EDDEEC.

\section{REFERENCES}

[1] A.Kashaf et al., "TSEP: Threshold-sensitive Stable Election Protocol for WSNs", 10th International Conference on Frontiers of Information 
Technology (FIT), 2012, pp.164-168.

[2] N.Javaid et al., "EDDEEC: Enhanced Developed Distributed Energy-Efficient Clustering for Heterogeneous Wireless Sensor Networks.", International Workshop on Body Area Sensor Networks, 2013, pp.914-919.

[3] M. Alshowkan et al., "LS-LEACH: A New Secure and Energy Efficient Routing Protocol for Wireless Sensor Networks", 17th International Symposium on Distributed Simulation and Real Time Applications (DS-RT), 2013, pp. 215-220.

[4] W.R. Heinzelman et al., "Energy-efficient communication protocol for wireless microsensor networks", in Proc. 33rd Hawaii IEEE International Conference on System Sciences, 2000, pp.1-10

[5] A. Manjeshwar and D. P. Agrawal., "TEEN: A Routing Protocol for Enhanced Efficiency in Wireless Sensor Networks", 1st International Workshop on Parallel and Distributed Computing Issues in Wireless Networks and Mobile Computing, 2001, pp.1-7.

[6] W. Heinzelman, A. Chandrakasan, and H. Balakrishnan, "An Application-Specific Protocol Architecture for Wireless Microsensor Networks," IEEE Transaction on Wireless Comm., vol. 1, no. 4, pp. 660- 670, 2002.

[7] Manjeshwar, Arati, and Dharma P. Agrawal. "APTEEN:A Hybrid Protocol for Efficient Routing and Comprehensive Information Retrieval in Wireless Sensor Networks.", Proceedings of the 16th International Parallel and Distributed Processing Symposium (IPDPS), 2002, pp.18.

[8] S. Lindsey and C. S. Raghavendra, "PEGASIS: Power-efficient gathering in sensor information systems", In Aerospace conference proceedings, 2002, pp. 1125-1130

[9] O. Younis and S. Fahmy, "HEED: A hybrid, energy-efficient, distributed clustering approach for ad hoc sensor networks", IEEE Transaction on Mobile Computing, vol.3, no.4, pp .366 -379, 2004.

[10] G. Smaragdakis, et al., "SEP: A Stable Election Protocol for clustered heterogeneous wireless sensor network", In Second International Workshop on Sensor and Actor Network Protocols and Applications (SANPA), 2004, pp.1-10.

[11] L.Qing et al., "Design of a distributed energy-efficient clustering algorithm for heterogeneous wireless sensor networks", Computer communications, vol. 29, no. 12, pp. 2230-2237, 2006.

[12] N.Israr and I.Awan, "Multihop clustering algorithm for load balancing in wireless sensor networks", International Journal of Simulation Systems, Science and Technology, vol. 8, no. 1, pp. 13-25, 2007.

[13] Md. Solaiman Ali et al., "ALEACH: Advanced LEACH routing protocol for wireless microsensor networks", In 5th International Conference on Electrical and Computer Engineering (ICECE), 2008, pp. 909-914.

[14] B.elbhiri et al., "Stochastic Distributed Energy-Efficient Clustering (SDEEC) for heterogeneous wireless sensor networks", ICGST-CNIR Journal, Vol.9, Dec, pp.11-17, 2009.

[15] B.Elbhiri et al., "Developed Distributed Energy-Efficient Clustering (DDEEC) for heterogeneous wireless sensor networks", 5th International Symposium on Communications and Mobile Network, 2010, pp.1-4.

[16] P.Saini and A.K.Sharma, "E-DEEC- Enhanced Distributed Energy Efficient Clustering Scheme for heterogeneous WSN", 1st International Conference on Parallel, Distributed and Grid Computing (PDGC), 2010, pp. 205-210

[17] S.B.ALLA at al., "Balanced and Centralized Distributed Energy Efficient Clustering for heterogeneous wireless sensor networks", 3rd International Conference on Next Generation Networks and Services, 2011, pp.39-44.

[18] Y.Miao et al., "Performance Study of Routing Mechanisms in Heterogeneous WSNs", International Conference on Computer Science \& Service System (CSSS), 2012, pp. 971-974.

[19] T.N. Qureshi et al., "On Performance Evaluation of Variants of DEEC in WSNs",7th International Conference on Broadband, Wireless Computing, Communication and Applications (BWCCA), 2012, pp.162-169.

[20] Khan, Abdul Gani. "A Comparative Analysis: Routing Protocols For Wireless Sensor Networks." MAIREC International Journal of Research in IT \& Management (IJRIM) 2 (2012): 516-534.

[21] Kashaf, Aasia, Nadeem Javaid, Zahoor Ali Khan, and Imran Ali Khan. "TSEP: threshold-sensitive stable election protocol for WSNs." arXiv preprint arXiv:1212.4092 (2012).(FOR ESEP)

[22] Mhatre, Vivek, and Catherine Rosenberg. "Homogeneous vs heterogeneous clustered sensor networks: a comparative study." In Communications, 2004 IEEE International Conference on, vol. 6, pp. 3646-3651. IEEE, 2004 